\title{
Disseminated aspergillosis following radiation therapy for medulloblastoma
}

\author{
P. Hamilton, P.J. Darbyshire, P. McKeever ${ }^{1}$ and A. Oakhill
}

Departments of Paediatric Haematology/Oncology and ${ }^{1}$ Paediatric Pathology, Bristol Children's Hospital, St. Michael's Hill, Bristol, UK.

\begin{abstract}
Summary: A 16 year old girl who underwent surgery and radiotherapy for medulloblastoma is described. Shortly following the completion of treatment symptoms developed suggestive of tumour recurrence. At post mortem the cause was identified as disseminated aspergillosis with extensive cerebral involvement. The importance of this unusual complication is discussed.
\end{abstract}

\section{Introduction}

Disseminated aspergillosis is usually found in the setting of the profoundly immunosuppressed patient, most commonly with acute leukaemia as an underlying diagnosis. We report a young girl in whom this complication arose following treatment for a medulloblastoma.

\section{Case report}

A 16 year old girl presented with a one year history of headache and a two month history of blurred vision in the right eye. She also complained of double vision on looking to the left.

Physical examination confirmed diplopia on left lateral gaze with a left VIth nerve palsy. Papilloedema was noted in the right eye. Computerized tomography (CT) of the brain revealed a space-occupying lesion displacing the fourth ventricle upwards. The lateral and third ventricles were distended. At operation, tumour was found in the lower part of the fourth ventricle extending downwards behind the brain and spinal cord to the level of the second cervical vertebra. The tumour was partially resected, complete removal being impossible. No shunt procedure was performed. The histological appearance of the tumour was characteristic of medulloblastoma.

Six weeks after the resection of the tumour the patient was transferred to the Bristol Children's Hospital and Bristol Radiotherapy Department for further treatment. At this time, she was still having

Correspondence: P.J. Darbyshire, Department of Haematology, Hospital for Sick Children, Great Ormond Street, London WC1N 1EH morning headaches, blurred vision and diplopia. A fluctuant swelling under the operation scar at the occiput was noted. A second CT scan of the brain showed a large collection of cerebrospinal fluid posteriorly, as well as enlarged and displaced ventricles unchanged from the previous scan. She was treated with dexamethasone $8 \mathrm{mg} / 6 \mathrm{~h}$. A course of radiotherapy was given with a maximum dose of 3660 centigray (cGy) to the head and $4465 \mathrm{cGy}$ to the spine. With top up dose, the total dose to the posterior fossa was 5500 cGy. Regular ketoconazole $(200 \mathrm{mg} / \mathrm{d})$ was given as prophylaxis against Candida infection.

Two weeks after starting radiotherapy and steroids she developed weakness of the legs. This was thought to be a steroid-induced myopathy and the dose of dexamethasone was gradually reduced to $2 \mathrm{mg} / 6 \mathrm{~h}$, but with minimal improvement. She became febrile, and since she was neutropenic received a 10 day course of ceftazidime, tobramycin and flucloxacillin. No pathogens, bacterial or fungal were found from blood, throat or urine cultures. A chest radiograph showed some patchy consolidation in both lung fields, but these changes completely resolved over the next few days.

Eight weeks after starting radiotherapy, she developed weakness of the proximal muscles of the right arm, progressing to total paresis of the arm two days later. It was feared that the tumour had extended or that she had developed metastases in the cervical spinal cord. A further CT scan of the head and neck, with contrast, however, showed no change. Two days later she developed paresis of the right leg. She continued to deteriorate and developed severe generalized weakness. She lapsed into a coma and died 10 weeks after starting radiotherapy.

At post mortem, the left kidney was found to be 
swollen and infarcted. There was a small area of infarction in the right lung. The left cerebral hemisphere was swollen with areas of softening and discolouration. Coronal slices revealed a lesion in the left frontoparietal region extending through the cortex and white matter to the ventricular wall. There were haemorrhagic and necrotic areas within the lesion (Figure 1). There were two similar but smaller lesions in the right cerebral hemisphere. The lesions did not correspond to any specific vascular distribution and appeared to be well established. A soft necrotic area in the cerebellar region, approximately $1 \mathrm{~cm}$ in diameter, appeared to be the only evidence of residual tumour.

Histological examination showed thrombi in some vessels of the lung and almost all vessels of the left kidney. These thrombi contained fungal hyphae typical of Aspergillus.

Histology of the brain showed an intense leptomeningeal infiltration by polymorphs and lymphocytes. The meningeal arteries and veins were distended by fibrin thrombi. Large numbers of fungal hyphae were present within the lumina of the vessels amongst the inflammatory infiltrate. The necrotic lesion in the left hemisphere was an area of recent infarction, with fungal hyphae present in the vessels and also spreading out into the nervous tissue.

The appearances were those of a meningo-encephalitis due to fungal infection with Aspergillus.

\section{Discussion}

Disseminated aspergillosis occurs almost exclusively in the immunocompromised patient, infection in the apparently immunocompetent is very rare but has however been reported (Cooper et al., 1981). In several large series (Degregorio et al., 1983; Fisher et al., 1981; Meyer et al., 1973; Young et al., 1970) of patients with malignancy and aspergillosis, leukaemia was the underlying diagnosis in $52 \%$. To our knowledge disseminated aspergillosis has not been recorded as a complication following therapy of a primary brain tumour. The reasons for the rarity of this fungal infection in these patients probably relates to the known factors predisposing to aspergillosis (Meyer $e t$ al., 1973). These include neutropenia, usually secondary to chemotherapy, previous broad spectrum antibiotic use, often with bacteraemia, and corticosteroid therapy. It is likely that only the latter factor operates in most brain tumour patients. However, our patient did have a short course of broad spectrum antibiotics for a febrile episode during a period of radiation induced neutropenia. She was also receiving high doses of steroids.

The portal of entry of the fungus is usually the lungs (Fisher et al., 1981), and evidence of primary infection in this site is found in $90 \%$ of cases, only $10 \%$ having

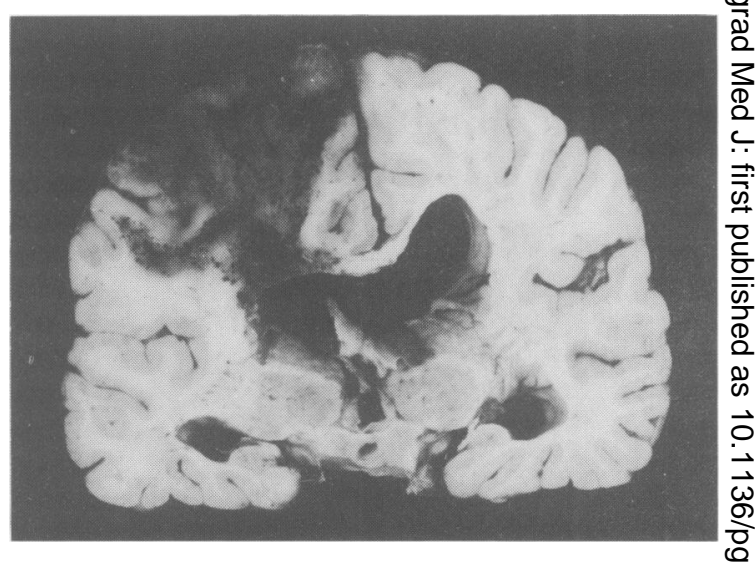

Figure I Coronal section of brain showing extensive infarc tion in the left frontoparietal region.

primary extrapulmonary disease. Our patient is dif- $\underset{\oplus}{\omega}$ ficult to classify in these terms, her cerebral lesions $c r$ were the most extensive but she did have a small pulmonary infarct. Her chest radiograph was abnor- $\rightarrow$ mal six weeks previously but at the time of her death $\frac{7}{0}$ showed no evidence of infection or infarction. It is possible that the primary infection was within the $\vec{\oplus}$ lungs but that cleared spontaneously as her immung on system recovered. It is unlikely that the cerebral lesions. were primary, acquisition at operation and subsequent survival for 3 months with cerebral aspergillosis would be most unusual.

Despite the unusual timing of her symptomatology, coming at the end of radiotherapy, we did not seriously consider fungal disease. Even had we done so the diagnosis would have been hard to establish. It is $\frac{3}{\sqrt{3}}$ usually made on bronchoscopy or lung biopsy of $\frac{7}{0}$ infected tissue, obviously impossible in this case with no evidence of pulmonary disease. Sputum examination is unreliable as a guide to invasive disease (Fisher et al., 1981), but a conversion of aspergillus serology 3 . may have been helpful. A CT scan performed a week $\delta$ before death failed to reveal any evidence of cerebral 3 abscess, infarct or tumour. It is possible that the large infarct in the left hemisphere had not yet developed, however recent evidence (Enzmann et al., 1980) suggests that the CT scan appearances of central nervous system fungal disease may be altered or absent in the $\widetilde{\sigma}$ immunosuppressed due to the poor immune response $N$ to the organism. Even in retrospect it appears that the diagnosis could only have been firmly established by a further craniotomy and biopsy. However examination of the cerebrospinal fluid may have suggested an inflammatory process.

Treatment of this infection is not in general successful. Ketoconazole, which this girl received as prophylaxis against Candida infection, does not have

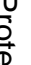


significant activity against Aspergillus, nor was it effective in preventing the disease in our patient. We have found only one case (Henze et al., 1982) of successful treatment of cerebral aspergillosis, this was achieved using amphotericin B, flucytosine and later surgical excision. Treatment of the more common pulmonary disease is slightly more successful. In one series (Fisher et al., 1981) 10 of 91 patients had their disease eradicated in the lungs using similar drugs. The major problem is in recognition of the disease before massive dissemination has occurred, as by that time treatment is usually unsuccessful.

This patient is unusual in developing disseminated aspergillosis as a complication of treatment for

\section{References}

COOPER, J.A.D., WEINBAUM, D.L., ALDRICH, T.K. \& MANDELL, G.L. (1981). Invasive aspergillosis of the lung and pericardium in a non immunocompromised 33 year old man. American Journal of Medicine, 71, 903.

DEGREGORIO, M.W., LEE, W.M.F., LINKER, C.A., JACOBS, R.A. \& RIES, C.A. (1983). Fungal infections in patients with acute leukaemia. American Journal of Medicine, 73, 543.

ENZMANN, D.R., BRANT-ZAWADZKI, M. \& BRITT, R.H. (1980). C.T. of central nervous system infections in immunocompromised patients. American Journal of Radiology, 135, 263.

FISHER, B.D., ARMSTRONG, D., YU, D. \& GOLD, J.W.M. medulloblastoma, the fungal disease mimicking tumour recurrence. It emphasises the need to consider fungal disease in any patient with malignancy however unlikely the setting, as only early recognition and aggressive treatment will improve the bleak outlook when this complication occurs.

\section{Acknowledgements}

We would like to thank Mr Torrance for permission to publish this case and Dr B. Brownell for performing the neuropathological examination. P.J.D. is supported by a grant from the Leukaemia Research Unit.

(1981). Invasive aspergillosis. American Journal of Medicine, 71, 571.

HENZE, G., ALDENHOFF, P., STEPHANI, U., GROSSE, G.A., KAZNER, E. \& STAIB, F. (1982). Successful treatment of pulmonary and cerebral aspergillosis in an immunosuppressed child. European Journal of Paediatrics, 138, 263.

MEYER, R.D., YOUNG, L.S., ARMSTRONG, D. \& YU, B. (1973). Aspergillosis complicating neoplastic disease. American Journal of Medicine, 54, 6.

YOUNG, R.C., BENNETT, J.E., VOGEL, C.L., CARBONE, P.P. \& DE VITA, V.T. (1970). Aspergillosis: The spectrum of disease in 98 patients. Medicine, 49, 147. 